\title{
Clinical and Postoperative Outcomes of Cancer Surgeries during the COVID-19 Pandemic: A Comparative Study with its Pre-COVID Surgical Audit at Five Major Tertiary Care Hospitals' Cancer Departments in India
}

\author{
Anil Heroor ${ }^{1}$ Rajnish Talwar ${ }^{2} \quad$ Rama Joshi $^{3} \quad$ Sandeep Nayak ${ }^{4} \quad$ B. Niranjan Naik ${ }^{5}$ Alok Tiwari ${ }^{5}$ \\ Sushil Kumar Jain ${ }^{5}$ Rajeev Kapoor ${ }^{2}$ Vijay Jagad ${ }^{2} \quad$ Naval Bansal $^{2}$ Bharath Gangadhara ${ }^{4}$ \\ Rashmi Bora ${ }^{3}$ Harish Verma ${ }^{5}$ Yadhukrishnan Nambiar ${ }^{1}$. Akshay Patil ${ }^{6}$ Hitesh Singhavi ${ }^{1}$, \\ ${ }^{1}$ Department of Surgical Oncology, Fortis Hospital, Mulund, \\ Maharashtra, India \\ 2Department of Surgical Oncology, Fortis Hospital Mohali, Punjab, \\ India \\ ${ }^{5}$ Department of Surgical Oncology, Fortis Memorial Research \\ Institute, Gurgaon, Haryana, India \\ 6Biostatistician, Tata Memorial Hospital, Mumbai, Maharashtra, \\ India
}

${ }^{3}$ Department of Gynae-Oncology Fortis Hospital, Gurugram, Haryana, India

${ }^{4}$ Department of Surgical Oncology, Fortis Cancer Institute, Bangalore, Karnataka, India
Address for correspondence Hitesh R. Singhavi, MDS, 1135, Fortis Hospital, Mulund, Maharashtra, India Pin Code - 4000012 (e-mail: hitsinx@gmail.com).

\begin{abstract}
Keywords

- pandemic

- COVID-19

- cancer surgery

- outcomes

- complication rates

Introduction There has been an exponential rise in number of coronavirus disease 2019 (COVID-19)-positive infections since March 23, 2020. However, cancer management cannot take a backseat.

Objective The aim of this study was to identify any difference in the complication and mortality rates for the cancer patients operated during the ongoing COVID-19 pandemic.

Materials and Methods This was a retrospective study of a prospectively maintained database of five centers situated in different parts of India. Variables such as demographics, intraoperative, and postoperative complications were compared between COVID-19 (group A-March 23, 2020-May 22, 2020) and pre-COVID time period (group B-January 1 to January 31, 2020).
\end{abstract}

DOI https://doi.org/ $10.1055 / \mathrm{s}-0041-1732858$ ISSN 0971-5851
(C) 2021. Indian Society of Medical and Paediatric Oncology. This is an open access article published by Thieme under the terms of the Creative Commons Attribution-NonDerivative-NonCommercial-License, permitting copying and reproduction so long as the original work is given appropriate credit. Contents may not be used for commercial purposes, or adapted, remixed, transformed or built upon. (https://creativecommons.org/licenses/by-nc-nd/4.0/). Thieme Medical and Scientific Publishers Private Ltd. A-12, Second Floor, Sector -2, NOIDA -201301, India 
Results One-hundred sixty-eight cancer surgeries were performed in group B as compared with 148 patients who underwent oncosurgeries in group A. Sixty-two percent lesser cancer surgeries were performed in the COVID-19 period as compared with the specific pre-COVID-19 period. There was no significant difference in age group, gender, comorbidities, and type of cancer surgeries. Except for the duration of surgery, all other intraoperative parameters like blood loss and intraoperative parameters were similar in both the groups. Minimally invasive procedures were significantly lesser in group A. Postoperative parameters including period of intensive care unit stay, rate of infection, need for the change of antibiotics, and culture growth were similar for both the groups. While minor complication like Clavien-Dindo classification type 2 was significantly higher for group A, all other complication rates were similar in the groups. Also, postoperatively no COVID-19-related symptoms were encountered in the study group. A subset analysis was done among the study groups between those tested preoperatively for COVID-19 versus those untested showed no difference in intraoperative and postoperative parameters. No health-care worker was infected from the patient during the time period of this study.

Conclusion Our study shows that there is no significant difference in the incidence of postoperative morbidity and mortality rates in surgeries performed during COVID-19 pandemic as compared with non-COVID-19 time period.

\section{Introduction}

The World Health Organization (WHO) declared coronavirus disease 2019 (COVID-19) as pandemic on March 11, 2020. ${ }^{1}$ In India, the first case was reported on January 30 , 2020. ${ }^{2}$ Since then there has been an exponential rise in the number of infected patients, crossing half a million by June end. At the onset of this pandemic, there was a national lockdown of 77 days starting on March 23. This resulted in complete paralysis of transport and all nonessential services. The Indian government has adapted the multiprong strategy to control the community spread of the virus. However, treatment of non-COVID-19 communicable and noncommunicable diseases took a backseat. The WHO estimates interruption in vaccination of 80 million infants due to this pandemic with Michigan study noting a significant decline in the vaccination drive in all the age groups of infants. ${ }^{3}$ According to the survey done among world emergency services members, there has been an $81.8 \%$ decrease in the total number of patients undergoing emergency surgery and $56.1 \%$ of the collaborators observed more severe septic abdominal diseases during the pandemic, especially for perforated appendicitis and cholecystitis (41.8 and $40.2 \%$, respectively) due to delay in diagnosis and intervention. ${ }^{4}$

Fear of acquiring the COVID-19 infection in hospital settings restricted patients to reaching health-care facilities. There was also an intense fear factor among health-care personnel regarding the risks involved in dealing with possible COVID-19 patients; these fears included high postoperative complication and mortality rates in COVID-19-positive patients, fear of acquiring infection, and bringing the infection home. Other countries where the infection was rampant were reporting higher mortality in general, and health-care personnel were getting infected leading to severe illness and at times death. ${ }^{5}$ All these factors led to a significant decrease in the number of patients being operated for cancer. ${ }^{6}$ Thus, we decided to conduct a multicentric observational study to look at the outcomes of surgeries during this period.

Table 1 Details of different centers of hospitals across India

\begin{tabular}{|l|l|l|l|l|}
\hline Centers & Group A & Group B & $\begin{array}{l}\text { COVID-19 test commencing } \\
\text { date }\end{array}$ & $\begin{array}{l}\text { Number of COVID-19 patients in the } \\
\text { state }\end{array}$ \\
\hline Mumbai & 61 & 57 & April 15 & 30,512 \\
\hline Bangalore & 22 & 45 & April 20 & 18,016 \\
\hline Mohali & 47 & 34 & April 20 & 114 \\
\hline Gurugram & 9 & 20 & April 20 & 5,463 \\
\hline $\begin{array}{l}\text { Gurugram } \\
\text { (Gynae) }\end{array}$ & 9 & 12 & April 20 & 5,463 \\
\hline
\end{tabular}


Our primary aim was to identify if there was any difference in the complication rates as well as mortality rates for the cancer patients operated during the pandemic. We also aimed to find out the infection rates of the health-care personnel involved in the care of these patients right from admission to discharge. These personnel included doctors, nurses, paramedical and other ancillary hospital staff involved in the care of these patients.

\section{Materials and Methods}

This was a retrospective study of prospectively maintained database of the five tertiary care centers (four centers of surgical oncology and a center of gynae-oncology) located in different parts of India: Mumbai, Bangalore, Gurugram, and Mohali. All patients (148 cancer patients) who underwent surgery for oncological indication from March 23, 2020 to May 22, 2020 were included in group A as study group. For the purpose of comparison, data of the patients (168 cancer patients) who underwent surgery indicated for cancer from January 1 to January 31, 2020 were included in group B. Thus, group A consisted of patients operated during COVID-19 pandemic and group B consisted of cancer patients operated during non-COVID-19 period. In total, 316 patients were included in the study. This group represented the control group as all these surgeries were done before the outbreak of COVID-19 pandemic in India.

All the adult patients above the age of 18, histopathologically proven cancer, indicated for surgery were included in the study. Patients with incomplete data including 11 patients from group A and 19 patients from Group B were excluded.

In the study group (group A), there were two subsets of patients; the first subset had no patients tested preoperatively for COVID-19 infection (from March 23, 2020 to April 15, 2020) and the second included patients who had been tested for COVID-19 infection. However, it varied according to the centers ( $\mathbf{- T a b l e ~} \mathbf{1}$ ). For the second subset, all the patients (11 patients) who were tested positive (reverse transcriptase-polymerase chain reaction [RT-PCR] positive) were not included in the study. All surgeries in group $A$, that is, the study group, were performed with all precautions including complete personal protective equipment's protocol. Data were manually collected from the electronic database and from the patients files extracted from the medical record department.

Preoperative parameters gathered and recorded included gender, age, Eastern Cooperative Oncology Group (ECOG), grade of surgery, comorbidities, chest X-ray, COVID-19 testing status, and primary site of cancer. Grade of surgery was categorized into four levels according to the complexity and technical difficulty of the surgery as defined by Lei et al. ${ }^{11}$ Intraoperatively parameters assessed included duration of surgery, blood loss, intraoperative injury if any, and type of anesthesia. Postoperative parameters including infection rate, change in antibiotics, prolonged antibiotics, and complication rates (Clavien-Dindo [CD] classification) ${ }^{7}$ were documented. Any symptoms similar to COVID-19 clinical presentation including fever, sore throat, fatigue, loss of taste or smell, nasal congestion, headache, muscle or joint pain, skin rash, nausea or vomiting, diarrhea, chills, or dizziness were also documented on subsequent follow-up to 15 days postoperatively. Laboratory confirmation of COVID-19 was done by quantitative RT-PCR on samples from the respiratory tract for all patients preoperatively in second subset of group $A$ and for all symptomatic postoperative patients. Postoperative surgical outcomes were the primary end-point, while change in the intraoperative parameters was secondary outcome.

\section{Data Accessibility Statement}

Data will be made available as per request.

\section{Statistical Analysis}

Demographic data were summarized with descriptive statistics. Continuous data was represented as mean (standard deviation) or median (interquartile range [IQR]) and categorical data was reported in counts (percentage). The Shapiro-Wilk test was used to check the normality of each variable. Descriptive analysis was performed to identify the distribution of variables under study. Comparison between the two groups with categorical demographic and clinicodemographic variables including gender, grade of surgery, primary site, intraoperative event, postoperative intensive care unit (ICU) stay, site of infection, complication rates, and culture was done using chi-squared test with Pearson's test. Mann-Whitney nonparametric test was used for comparison of continuous demographic and clinicodemographic variables including age, duration of surgery, blood loss, and hospital stay. For all tests, $p$-values were two-sided, and a $p$-value lower than 0.05 was considered statistically significant. Statistical analyses were performed using SPSS software (released 2017; IBM SPSS Statistics for Windows, Version 25.0. Armonk, NY: IBM Corp).

\section{Ethics}

The study protocol was presented and approved by Fortis institutional ethics committee (IEC) on June 7, 2020 and waiver of informed consent was obtained (IEC/2020/OAS/04). The procedures followed were in accordance with the ethical standards of the responsible committee on human experimentation (institutional) and with the Helsinki Declaration of 1964 , as revised in 2013.

\section{Results}

One-hundred sixty-eight cancer surgeries were performed in group B as compared with 148 patients who underwent oncosurgeries in group A ( - Table 2). Sixty-two percent lesser cancer surgeries were performed in the COVID-19 period as compared with the specific pre-COVID-19 period (-Fig. 1). The median age of the patients in group A was 55.5 years (IQR: 42.25-64.00), while that of group B control group was 57 years (IQR: 47-65). There was no significant difference in the median age of the patients operated between the groups ( $p=0.173)$. Group B $(44.6 \%)$ had more patients above the age of 60 years as compared with group A (37.2\%); however, it did not achieve statistical significance $(p=0.178)$. 
314 Cancer Surgical Complication Rates during COVID-19 Pandemic Heroor et al.

Table 2 Comparison of demographics and outcomes (intraoperative and postoperative) between two time periods

\begin{tabular}{|c|c|c|c|}
\hline Clinical characteristics & Before COVID-19, $n(\%)$ & During COVID-19, $n(\%)$ & $p$-Value \\
\hline \multicolumn{4}{|l|}{ Gender } \\
\hline Female & $113(67.3 \%)$ & 99 (66.9\%) & 0.944 \\
\hline Male & $55(32.7 \%)$ & $49(33.1 \%)$ & \\
\hline \multicolumn{4}{|l|}{ Grade of surgery } \\
\hline Grade 1 & $48(28.6 \%)$ & $36(24.3 \%)$ & 0.198 \\
\hline Grade 2 & $38(22.6 \%)$ & $34(23 \%)$ & \\
\hline Grade 3 & $55(32.7 \%)$ & $63(42.6 \%)$ & \\
\hline Grade 4 & $27(16.1 \%)$ & $15(10.1 \%)$ & \\
\hline \multicolumn{4}{|l|}{ Comorbidity } \\
\hline No & $78(46.4 \%)$ & $69(46.6 \%)$ & 0.854 \\
\hline One comorbidity & $52(31 \%)$ & $49(33.1 \%)$ & \\
\hline More than 2 comorbidities & $38(22.6 \%)$ & $30(20.3 \%)$ & \\
\hline \multicolumn{4}{|l|}{ Primary site } \\
\hline Head and neck & $34(20.2 \%)$ & $24(16.2 \%)$ & 0.023 \\
\hline Breast & $47(28 \%)$ & $43(29.1 \%)$ & \\
\hline Gastrointestinal & $42(25 \%)$ & $28(18.9 \%)$ & \\
\hline Gynae-oncology & $19(11.3 \%)$ & $23(15.5 \%)$ & \\
\hline Others & $9(5.4 \%)$ & $7(4.7 \%)$ & \\
\hline Uro and nephro-oncology & $2(1.2 \%)$ & $6(4.1 \%)$ & \\
\hline Chemoport procedures & $15(8.9 \%)$ & $17(11.5 \%)$ & \\
\hline \multicolumn{4}{|l|}{ Intraoperative events } \\
\hline Yes & $2(1.2 \%)$ & $4(2.7 \%)$ & 0.326 \\
\hline No & $166(98.8 \%)$ & $144(97.3 \%)$ & \\
\hline \multicolumn{4}{|l|}{ Surgery } \\
\hline OPEN & $92.6 \%$ & $7.4 \%$ & $<0.0001$ \\
\hline Minimal invasive & $77.4 \%$ & $22.6 \%$ & \\
\hline \multicolumn{4}{|l|}{ Postoperative ICU stay } \\
\hline Yes & $11(6.5 \%)$ & $13(8.8 \%)$ & 0.453 \\
\hline No & $157(93.5 \%)$ & $135(91.2 \%)$ & \\
\hline \multicolumn{4}{|l|}{ Surgical site infection } \\
\hline Yes & $13(7.7 \%)$ & $10(6.8 \%)$ & 0.738 \\
\hline No & $155(92.3 \%)$ & $138(93.2 \%)$ & \\
\hline \multicolumn{4}{|l|}{ Change of antibiotics } \\
\hline Yes & $17(10.1 \%)$ & $10(6.8 \%)$ & 0.286 \\
\hline No & $151(89.9 \%)$ & $138(93.2 \%)$ & \\
\hline \multicolumn{4}{|l|}{ Complication } \\
\hline CD1 & $3(1.8 \%)$ & $9(6.1 \%)$ & 0.038 \\
\hline CD2 & $20(11.9 \%)$ & $31(20.9 \%)$ & \\
\hline CD3A & $2(1.2 \%)$ & $0(0 \%)$ & \\
\hline CD3B & $1(0.6 \%)$ & $2(1.4 \%)$ & \\
\hline
\end{tabular}


Table 2 (continued)

\begin{tabular}{|l|l|l|l|}
\hline Clinical characteristics & Before COVID-19 $n$ (\%) & During COVID-19 $n(\%)$ & $p$-Value \\
\hline None & $141(83.9 \%)$ & $105(70.9 \%)$ & $1(0.7 \%)$ \\
\hline CD4 & $1(0.6 \%)$ & & \\
\hline Culture & & $4(2.7 \%)$ & 0.390 \\
\hline Growth & $8(4.8 \%)$ & $144(97.3 \%)$ & \\
\hline No growth & $160(95.2 \%)$ & $\begin{array}{l}\text { During COVID-19 } \\
\text { Median (IQR) }\end{array}$ \\
\hline & $\begin{array}{l}\text { Before COVID-19 } \\
\text { Median (IQR) }\end{array}$ & 5.173 \\
\hline Age (years), median (IQR) & $57(47-65)$ & $55.50(42.25-64)$ & 0.023 \\
\hline Duration of surgery(minutes), median (IQR) & $120(60-227.50)$ & $90(48.75-180)$ & 0.706 \\
\hline Blood loss(mL), median(IQR) & $30(10-100)$ & $30(15-100)$ & 0.815 \\
\hline Hospital stay(d), median(IQR) & $3.00(2.00-6.00)$ & $4(2-5)$ & \\
\hline
\end{tabular}

Abbreviations: CD, Clavien-Dindo classification used for complication rates; COVID-19, coronavirus disease 2019; ICU, intensive care unit; IQR, interquartile range.

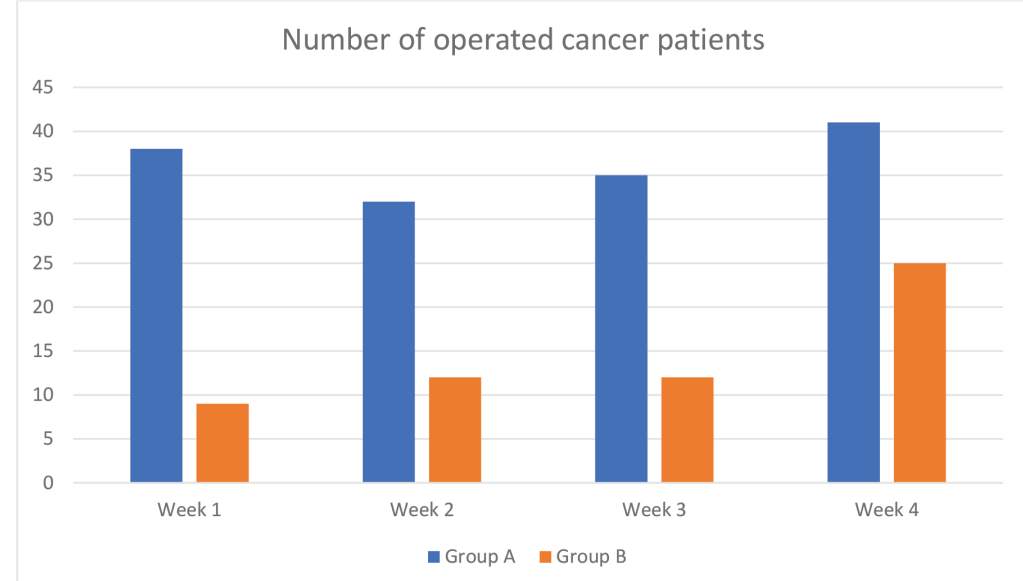

Fig. 1 Comparison of number of cancer patients operated in first 4 weeks between two time periods (group A vs. group B).

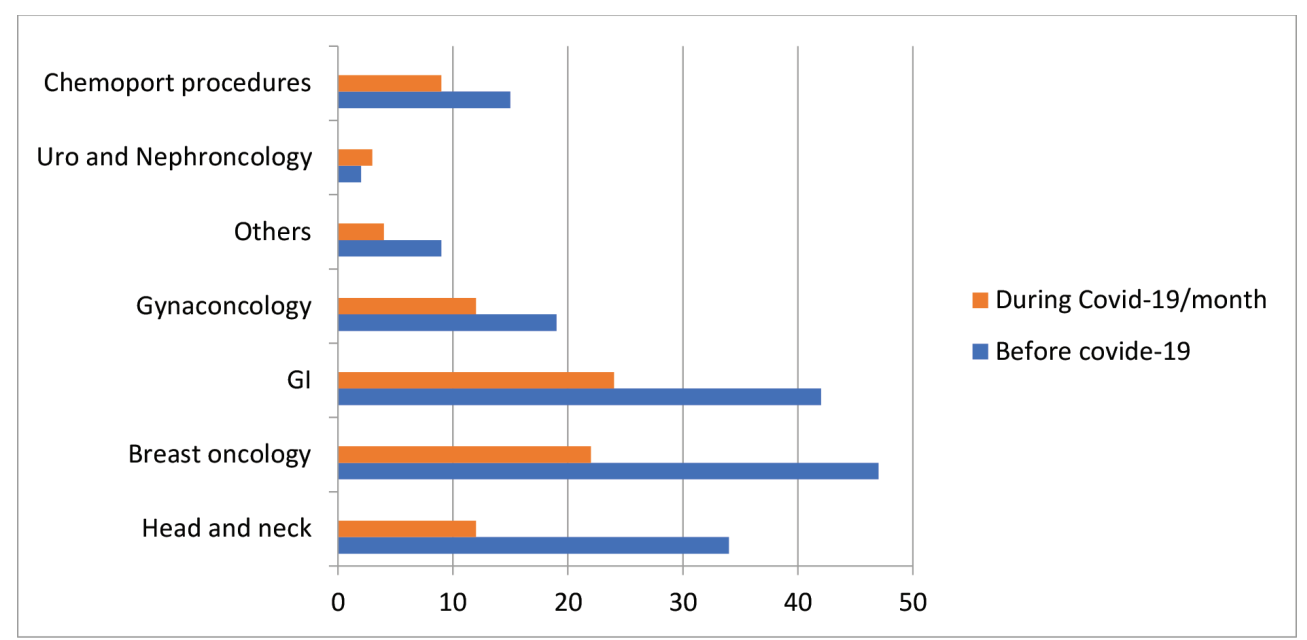

Fig. 2 Comparison of site-specific surgeries in coronavirus disease 2019 (COVID-19) versus non-COVID pandemic. GI, gastrointestinal.

Females were in majority in both the time periods (group A vs. group B; 66.9 vs. $67.3 \% ; p=0.944$ ). Grade of surgery was classified into four categories. Both the groups (group A and group B) had a similar percentage of the grade of surgeries $(p=0.282)$ (-Table 2). There was no significant difference in the percentage and type of comorbidities in both the groups 


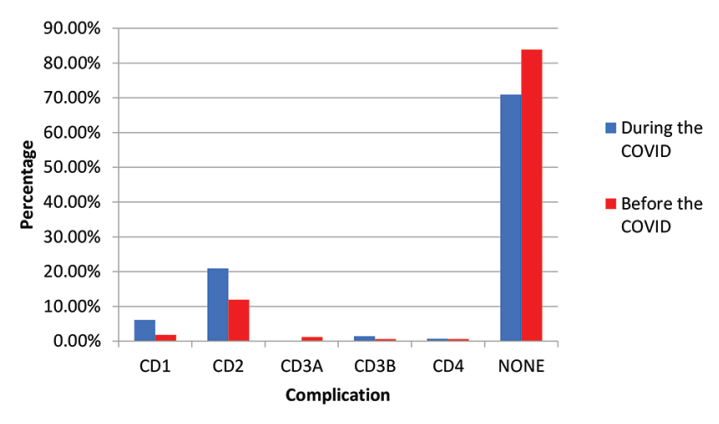

Fig. 3 Comparison of complication of cancer surgery patient in coronavirus disease 2019 (COVID-19) versus non-COVID-19 pandemic.

(0.832). Major site of cancer surgeries was similar in both the groups. Breast cancer surgery (28.5\%) was the most common followed by gastrointestinal surgeries (22.2\%), head and neck surgeries (18.4\%), gynae-oncology (13.3\%), uro-oncology, and chemoport insertion procedures. There were significantly lower cancer surgeries in group A for all anatomical sites (-Fig. 2).

Majority of patients in both groups had normal chest X-ray findings (97.3 vs. 96.4\%). Four patients had an abnormal chest X-ray in group A. Three of them did not have any postoperative complication, while one patient had $\mathrm{CD}-2$ type complication. However, all patients (five) in group B had no postoperative complications. There was no change in intraoperative events among both the groups. $(p=0.285)$. Minimally invasive procedures (including laparoscopic surgeries and robotic surgeries) were performed significantly less in COVID-19 period as compared with pre-COVID-19 period (7.4 vs. $22.6 \%$ ). The median duration of the operative procedures was significantly less in group A as compared with group B (90.00 min [IQR: $48.75-180.00 \mathrm{~min}$ ] vs. 120.00 min [IQR: $60.00-227.50 \mathrm{~min}$ ]; $p=0.023$ ). There was no significant difference in blood loss among the two study groups (30.00 mL [IQR: $15.00-100.00 \mathrm{~mL}$ ] vs. $30.00 \mathrm{~mL}$ [IQR: 10.00$100.00 \mathrm{~mL}] ; p=0.706$ ).

The median length of hospital stay was similar in both the study groups (group A vs. group B; 4.00 days [IQR: 2.00-5.00] vs. 3.00 days [IQR: $2.00-6.00$ ]; $p=0.815$ ). Postoperative ICU stay ( 8.8 vs. $6.5 \% ; p=0.453$ ), postoperatively infection rate (6.8 vs. $7.7 \% ; p=0.526$ ), and rate of change of antibiotics ( $6.8 \mathrm{vs.}$ $10.1 \% ; p=0.319$ ) were similar in both the groups. However, there was significant difference in CD-2 complication rates (20.3 vs. $11.9 \%$; - Fig. 3 ). There was no difference in the presence of bacterial or fungal infection in postoperative blood culture investigation among the study groups (2.7 vs. $4.8 \%$; $p=0.390$ ).

Preoperative COVID-19 testing was done in $68.24 \%$ of the group A and all were negative. However, 31.76\% never underwent testing. Therefore, we did a subgroup analysis in group A for those patients undergoing COVID-19 testing versus those who did not undergo testing. There was no significant difference in intraoperative events, blood loss, and duration of surgery in this subgroup analysis. We also did not find any significant difference in any parameters of postoperative complication including infection, change of antibiotics, culture growth, and complication rates in term of CD classification. No health-care worker was infected in direct association with surgery or postoperative care.

\section{Discussion}

COVID-19 disease has intensely stretched the health-care system to a breakpoint. With more than 700,000 COVID-19 cases by end-June 2020, India ranks fourth in the total number of COVID-19 infection across the world. ${ }^{8}$ Though our death rates (10 per million population) have been below the global rates (59.6 per million population), we still are away from our peak incidence of infection. ${ }^{8}$ Globally, it has been estimated that 90,000 health workers were infected till mid-May 2020. ${ }^{9}$ Both our frontline workers and hospital system are already overwhelmed with COVID-19-positive patients and treatment of non-COVID-19 disease has taken a serious toll. We do not have sufficient studies to indicate the outcome of delays in cancer surgeries. Approximately 2,100 Indians die every day due to cancer. ${ }^{10}$ These numbers would certainly increase due to straining of already resource constraints system and synergistic effects of COVID-19 infection. Restrictive measures like national lock down and home quarantine measures have certainly helped in taming the infection and flattening the curve. But it had its own side effects on the health-care system including the difficulty faced by the patients to reach hospital and additional health-care workers being allocated to manage the COVID-19 burden.

Therefore, we decided to assess whether COVID-19 infection and the change in the way of management of cancer patients in resource constraint settings would have any effects on treatment and postoperative complication of cancer patients. Three-hundred sixteen cancer patients were operated at five different tertiary care centers situated in different parts of India. One-hundred forty-eight cancer patients were operated during COVID-19 (2 months) as compared with 168 patients during non-COVID-19 period (1 month). A study led by Lei et al published in April 2020 found a higher mortality rate of $20.6 \%$ in asymptomatic COVID-19-positive patients who were in their incubation period during surgery. ${ }^{11}$ Another study that analyzed 1,128 patients showed mortality rate of $23.9 \%$ during COVID-19 period; however, it should be noted that preoperative positive severe acute respiratory syndrome coronavirus 2 (SARS-CoV-2) undergoing elective surgeries had lower mortality rate $(9.1 \%)$ as compared with those patients who were infected in perioperative settings (50\%). ${ }^{12}$ With evolving evidence against elective surgeries and all the global guidelines favoring only emergency surgeries, $62 \%$ lesser cancer surgeries were performed in the COVID-19 period as compared with the specific pre-COVID-19 period. It had been estimated that there would be $72.3 \%$ cancellation of surgery world over during 12 -week lockdown period with total cancellation of surgeries accounting to 28 million procedures. ${ }^{13}$ However, a study led by Shrikhande et al demonstrated no mortalities in cancer 
patients operated during COVID-19 period at a tertiary care center in Mumbai. In this study, only 23.7\% were tested preoperatively for COVID-19. ${ }^{6}$ Similarly, we also had 41 cancer patients operated, who were not tested for COVID-19. In our study, we also did not encounter any mortality during the COVID-19 period. All patients were screened for symptoms of COVID-19 in their follow-up consultation. Time period of our study was seeing the surge in COVID-19 cases where one of the centers was located (Mumbai). In such situation as well, we had $31.6 \%$ cases who were not tested for COVID-19. However, we did not have any COVID-19-positive patient even in the postoperative period. While majority of the centers participating in the study had dedicated COVID-19 wards and non-COVID-19 ICUs in their institute, results show that they were successful in managing the COVID-19 burden without inflicting hospital-acquired COVID-19 infection to the admitted non-COVID-19 patients. Age group operated during COVID-19 was similar to pre-COVID-19 period. Even in COVID-19 pandemic, approximately more than half of the surgeries were grade 3 and grade 4, which were proportionately more than pre-COVID-19. Therefore, technically challenging surgeries did not take a backseat even during these testing times.

Site-wise distribution of cancer surgeries represents the chronological incidence of cancer in India. Breast cancer surgeries were the most common operative procedures. Females outnumbered males in the number of operative procedures in COVID-19 pandemic. Comorbidities are highly associated with high mortality rate in SARS-CoV-2-infected patients and especially those undergoing surgeries. ${ }^{14}$ In our study, more than half of the patients undergoing cancer surgeries had presence of one or more comorbidities that was similar for both the groups. Intraoperative parameters like intraoperative complications were similar in both the groups. However, duration of surgery during COVID-19 period was much shorter. This could be attributed to careful case selection during this period or less minimal invasive surgeries as these surgeries do take a little more time as compared with open surgeries. Due to global guidelines against the use of laparoscopic instrumentation and use of robot during pandemic, there was significantly less usage of such minimal invasive technique. ${ }^{15}$

According to the need of the hour, we expected the surgeons to operate those patients who would not require postoperative ICU care. However, hospital infrastructure was equipped enough to handle such situations. We did not find any significant difference in postoperative ICU admissions as compared with non-COVID-19 period. Postoperative parameters including infection rate, blood culture growth, and change of antibiotics were individually similar in both the groups. However, complication rate (CD classification) was higher in COVID-19 period. When subgroup analysis of CD classification was done, there was significant difference in $C D-2$, while all other types had a similar outcome. This could be attributed to higher change in pharmacological management due to cumulative higher infection rate, usage of higher antibiotics, and fever in postoperative period. However, all patients were discharged uneventfully except one patient. One patient took discharge against the medical advice on the fourth day. Average length of stay was similar for both the groups. Data collected for the first two follow-ups after the surgery showed no COVID-19-related symptoms. These two follow-up visits were within the first 15 days after surgery in most of the patients. No patient underwent COVID-19 testing in postoperative phase. Thus, follow-up data concluded that there was no hospital acquired infection in the study group. No health-care worker directly involved in the patient care in the study group tested positive for COVID-19 in the stipulated time period.

\section{Novelty}

This study is a unique collaborative work of five centers situated in different parts of India and thus results of this study are representative of the impact of COVID-19 on cancer surgeries in hospitals across India. This is the first study to compare perioperative morbidity in COVID-19 pandemic compared with its own dataset of non-COVID-19 pandemic thus significantly reducing the influence of confounding factors.

\section{Limitation}

It involves all the potential biases included in a retrospective review. Results are not applicable to laboratory-confirmed COVID-19-positive cancer patients. Different parts of India were having surges of COVID-19 infection at variable times; however, multicentric nature of the study truly depicts the true status of major urban cities.

\section{Implication}

Hospital administration should have strategies in place to commence routine cancer surgeries in near future with the environment that remains exposed to SARS-CoV-2. This will help reduce the in-hospital transmission of SARS-Cov-2 without escalating to more postoperative complications. Therefore, consideration should be given on development of guidelines to avoid delaying or cancelling the operative procedures related to cancer surgeries even during the pandemic.

\section{Conclusion}

Non-COVID-19 cancers patients should be treated with the same vigor as they are associated with high mortality rate if left untreated. Our study shows that with proper planning, training of the personnel and dedicated COVID-19 and non-COVID-19 staff, and usage of the stipulated personal protective equipment (PPE) kits, it is possible to treat the non-COVID-19 diseases without any significant increase in postoperative complication rates.

\section{Funding \\ None. \\ Conflict of Interest \\ None.}




\section{Acknowledgments}

Authors would like to thank Dr Vasundhara Atre, Dr Gaurav and Dr Pooja Bora for all the technical assistance.

\section{Reference}

1 Cucinotta D, Vanelli M. WHO declares COVID-19 a pandemic. Acta Biomed 2020;91(1):157-160

2 Andrews MA, Areekal B, Rajesh KR, et al. First confirmed case of COVID-19 infection in India: a case report. Indian J Med Res 2020;151(5):490-492

3 Bramer CA, Kimmins LM, Swanson R, et al. Decline in child vaccination coverage during the COVID-19 pandemic Michigan Care Improvement Registry, May 2016-May 2020. Am J Transplant 2020;20(7):1930-1931

4 Reichert M, Sartelli M, Weigand MA, et al. WSES COVID-19 emergency surgery survey collaboration group. Impact of the SARS-CoV-2 pandemic on emergency surgery services-a multi-national survey among WSES members. World J Emerg Surg 2020;15(1):6410.1186/s13017-020-00341-0

5 Tabish SA. COVID-19 pandemic: Emerging perspectives and future trends. J Public Health Res 2020;9(1):178610.4081/ jphr.2020.1786

6 Shrikhande SV, Pai PS, Bhandare MS, et al. all collaborators from Department of Surgical Oncology. Outcomes of elective major cancer surgery during COVID 19 at Tata Memorial Centre: implications for cancer care policy. Ann Surg 2020;272(3):e249-e252

7 Clavien PA, Barkun J, de Oliveira ML, et al. The Clavien-Dindo classification of surgical complications: five-year experience. Ann Surg 2009;250(2):187-196

8 Matta S, Chopra KK, Arora VK. Morbidity and mortality trends of Covid 19 in top 10 countries. Indian J Tuberc 2020;67(4S): S167-S172
9 Bhadra A, Mukherjee A, Sarkar K. Impact of population density on Covid-19 infected and mortality rate in India. [published online ahead of print, 2020 Oct 14] Model Earth Syst Environ 2020. DOI: 10.1007/s40808-020-00984-7

10 Dikshit R, Gupta PC, Ramasundarahettige C, et al; Million Death Study Collaborators. Cancer mortality in India: a nationally representative survey. Lancet 2012;379(9828):1807-1816

11 Lei S, Jiang F, Su W, et al. Clinical characteristics and outcomes of patients undergoing surgeries during the incubation period of COVID-19 infection. EClinicalMedicine 2020;21:100331, https://www.thelancet.com/journals/eclinm/article/PIIS25895370(20)30075-4/abstract cited2020Jul5 [Internet]

12 Mortality and Pulmonary Complications in Patients Undergoing Surgery With Perioperative SARS-CoV-2 Infection: An International Cohort Study [Internet]. Lancet (London, England). 2020 [cited 2020 Jun 21]. Available from: https:// pubmed.ncbi.nlm.nih.gov/32479829/. Accessed July 4, 2021

13 Elective Surgery Cancellations Due to the COVID-19 Pandemic: Global Predictive Modelling to Inform Surgical Recovery Plans [Internet]. The British journal of surgery. 2020 [cited 2020 Jun 21]. Available from: https://pubmed.ncbi.nlm.nih. gov/32395848/. Accessed July 4, 2021

14 Guan WJ, Liang WH, Zhao Y, et al. China Medical Treatment Expert Group for COVID-19. Comorbidity and its impact on 1590 patients with COVID-19 in China: a nationwide analysis. Eur Respir J 2020;55(5):2000547, Available on: https://www. ncbi.nlm.nih.gov/pmc/articles/PMC7098485/ cited2020Jul5 [Internet] Accessed on September 25, 2021

15 Khan J, van Boxel G, Mercer S. Is minimal access surgery possible and safe during the COVID-19 pandemic? $\mathrm{Br}$ J Surg 2020;107(8):e268 